\section{RICYDE. Revista Internacional de Ciencias del Deporte} doi: $10.5232 /$ ricyde

Rev. int. cienc. deporte

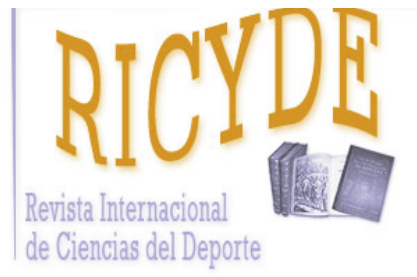

RICYDE. Revista Internacional de Ciencias del Deporte VOLUMEN XII - AÑO XII

Páginas:359-381 ISSN:1885-3137

Número 46 - Octubre - 2016

\title{
Pedaleo de brazos en personas con lesión medular, parálisis cerebral o ataxia cerebelosa: Parámetros fisiológicos Armcrank pedaling in persons with spinal cord injury, cerebral palsy or cerebellar ataxia: Physiological parameters
}

\author{
Iris González-Carbonell1 ${ }^{1}$ Gabriel Brizuela2 ${ }^{2}$ José Luis Romero-Ávila3
}

1. Universidad Internacional de Valencia. España

2. Departamento de Educación Física y Deportiva, Universidad de Valencia. España

3. Departamento de Fisioterapia, Universidad de Valencia.España

\section{Resumen}

Los desórdenes neurológicos generan afectación física y derivan en sedentarismo, enfermedades coronarias y obesidad o diabetes, reduciendo tanto la esperanza como la calidad de vida. La oferta de actividad física adaptada es escasa por falta de información específica sobre la forma de adecuarlo y dosificarlo a las personas que presentan estos desórdenes. Con el fin de comparar el efecto del ejercicio de pedaleo de brazos sobre la respuesta fisiológica y la percepción del esfuerzo, en 8 personas con lesión medular, 4 con parálisis cerebral y 4 con ataxia de Friedreich, se analizó su respuesta fisiológica, así como su percepción subjetiva al esfuerzo, frente a un grupo Control (16 participantes). Para ello realizaron un ejercicio normalizado de pedaleo de brazos en un ergómetro y se midieron frecuencia cardíaca, frecuencia respiratoria, volumen corriente, volumen espirado, consumo de Oxígeno relativo, pulso de Oxígeno y percepción del esfuerzo. Se realizó un ANOVA con estas variables. Para el grupo con lesión medular, el estrés y gasto energético resultaron los más bajos. El grupo con parálisis cerebral mostró los niveles de estrés más altos, además percibiéndolo como una carga moderada. El grupo con ataxia de Friedreich, mostró respuestas cardiorrespiratorias altas intermedias. Se puede concluir que diferentes desórdenes neurológicos muestran respuestas fisiológicas muy diferentes frente al ejercicio y es importante su control.

Palabras clave: ejercicio; actividad física; lesión medular; ataxia; parálisis cerebral.

\section{Abstract}

Neurological disorders produce physical impairment that result in physical inactivity, heart disease and obesity or diabetes, reducing both life expectancy and quality of life. The supply of adapted physical activity is limited by lack of specific information on how to adapt and dose to people who have these disorders. In order to compare the effect of armcrank pedaling exercise on their physiological response and perceived exertion, 8 persons with spinal cord injury, 4 with cerebral palsy and 4 with Friedreich's ataxia were analyzed, comparing their physiological responses as well as their perceived effort, to a control group (16 participants). They performed a standardized armcrank pedaling exercise on an ergometer and heart rate, respiratory rate, tidal volume, expiratory volume, relative oxygen consumption, oxygen pulse and perceived exertion were measured. An ANOVA test was performed with these variables. For the group with spinal cord injury, stress and energy expenditure were the lowest, the group with cerebral palsy showed the highest levels of stress while perceived it as a moderate load. The group with Friedreich's ataxia showed medium high cardiorespiratory responses. In conclusion, it has been identified as different neurological disorders show very different physiological responses to exercise and the importance of their control.

Key words: exercise; physical activity; spinal cord injury; ataxia; cerebral palsy. 
González-Carbonell, I.; Brizuela, G., y Romero-Ávila, J. L. (2016). Pedaleo de brazos en personas con lesión medular, parálisis cerebral o ataxia cerebelosa: Parámetros fisiológicos. RICYDE. Revista internacional de ciencias del deporte. 46(12), 359-381. http://dx.doi.org/10.5232/ricyde2016.04602

\section{Introducción}

$\mathrm{D}$ esde hace décadas, existe suficiente evidencia científica para asegurar que la práctica de actividad física (AF) de forma regular genera beneficios tanto a nivel físico, como psicológico y social (Folkins y Sime, 1981; Malina y Bouchard, 1991; Devís-Devís, 2001). Por el contrario, la inactividad aumenta notablemente las posibilidades de tener sobrepeso, obesidad, enfermedades cardiovasculares y respiratorias, trastornos crónicos como la diabetes, enfermedades musculo-esqueléticas o incluso desórdenes psicológicos (Lawson, 2006). Esto se cumple incluso para las personas con discapacidad de tipo físico, quienes frecuentemente llevan una vida más sedentaria, con una disminución de su condición física, (Verschuren y Takken, 2010), un incremento de su masa grasa, reducción de su masa muscular, y la muy frecuente aparición de los mencionados problemas de salud (Hjeltnes y Wallberg-Henriksson, 1998), incluso en un detrimento de su estatus funcional, su participación, su autonomía y finalmente su calidad de vida (Haisma y col., 2006).

La lesión medular (LM) y la parálisis cerebral (PC) son dos desórdenes neurológicos de prevalencia relevante y origen frecuente de discapacidad física. Junto a la ataxia de Friedreich (ADF), de menor prevalencia que las anteriores, estos tres desórdenes neurológicos podrían sumar una incidencia total de entre 1,60 y 2,60 casos por cada 1.000 habitantes de la población mundial (Toung y col., 2004; Bax y col., 2005; Delatycki, Williamson y Forrest, 2000).

La LM es una alteración de la medula espinal que provoca pérdida de sensibilidad y/o movilidad. Se define como "Tetraplejia" a la pérdida de función motora y/o sensitiva por una lesión en los segmentos cervicales de la médula espinal que ocasiona un déficit funcional tanto en los brazos, tronco como en piernas y órganos pélvicos" (Ditunno, Young, Donovan y Creasey, 1994). El $66 \%$ de estas personas tiene sobrepeso u obesidad (Rajan. McNeely, Warms y Goldstein, 2008) y el colectivo presenta una mortalidad prematura. Las neumonías son la primera causa de mortalidad, seguido de las enfermedades cardiovasculares y las enfermedades urogenitales (Lidal y col., 2007), así como una tendencia a una mayor hipertensión y enfermedades cardiovasculares (Krum y col., 1992), siendo la práctica habitual de AF, una buena alternativa para reducir el riesgo de enfermedades cardiovasculares (Washburn y Figoni, 1999; Brenes, Dearwater, Shapera, LaPorte y Collins, 1986).

Según Bax y col. (2005) la PC se define como "un conjunto de cambios en el desarrollo del movimiento y de la postura, causadas por alteraciones no progresivas del sistema nervioso central (cerebro, cerebelo y tronco cerebral) durante la maduración del mismo, que provoca limitaciones en la actividad". Estas limitaciones en la actividad vienen determinadas por la movilidad reducida y la postura adquirida (Jahnsen, Villien, Egeland, Stanghelle y Holm, 2004; Thorpe, 2009; Mutch, Alberman, Hagberg, Kodama y Perat, 1992). El paso del tiempo acentúa el deterioro de su condición física y también es frecuente el dolor crónico, la fatiga o la deformación de las articulaciones, incluso la mortalidad prematura, asociada al sedentarismo (Murphy, Molnar y Lankasky, 1995; Engel, Schwartz, Jensen y Johnson, 2000; Peterson y col., 2013 b; Bauman, 2009). Sin duda, la práctica regular de AF resultará determinante para mantener o mejorar su calidad de vida (Erikssen y col., 1998; Taylor, Dodd y Larkin, 2004; Bauman, 2009; Peterson, Gordon y Hurvitz, 2013 a).

La ADF es un trastorno neurodegenerativo, de origen genético, que causa un deterioro progresivo de muchas funciones (Chamberlain y col., 1988), afectando la coordinación en los movimientos multiarticulares, al equilibrio y al control postural (Bastian, 1997; Mariotti, Fancellu y Di Donato, 2005). Las miocardiopatías y la diabetes mellitus son las 
González-Carbonell, I.; Brizuela, G., y Romero-Ávila, J. L. (2016). Pedaleo de brazos en personas con lesión medular, parálisis cerebral o ataxia cerebelosa: Parámetros fisiológicos. RICYDE. Revista internacional de ciencias del deporte. 46(12), 359-381. http://dx.doi.org/10.5232/ricyde2016.04602

complicaciones más extendidas y muy posiblemente relacionadas con el sedentarismo (Delatycki y col., 2000; Ristow y col., 1998; Maring y Croarkin, 2007). Apenas existen trabajos que analicen el efecto de la AF sobre su condición física, destacando un estudio de Fillyaw y Ades (1989) en el que detectaron mejoras tanto de su capacidad como de su potencia aeróbica, tras 27 sesiones de entrenamiento aeróbico, tras 27 sesiones de entrenamiento aeróbica de $20 \mathrm{~min}$ en cicloergómetro. Posteriormente, Ilg y col. (2009) encontraron mejorías en la velocidad de marcha, así como en el balanceo y la coordinación de las piernas, tras un entrenamiento de la coordinación.

Desde un punto de vista energético, las personas que se desplazan habitualmente en silla de ruedas muestran que tienen un gasto energético (GE) mucho menor que el resto de la población (Dearwater, Laporte, Cauley y Brenes, 1985; Conger y Basset, 2011; Monroe y col., 1998; Collins y col., 2010). Este gasto es aún más reducido en personas con niveles de afectación física mayor, por ejemplo, con LM cervical (Yamasaki, Irizawa y Komura, 1992; Buchholz y Pencharz, 2004). En estudios con personas adultas con PC (Johnson, Hildreth, Contompasis y Goran, 2005) se recalca que existe gran variabilidad energética entre las personas deambulantes y las que no. Finalmente, en personas con ADF, la mutación genética provoca alteraciones en la producción energética mitocondrial y se sugiere una menor eficiencia aeróbica así como un deterioro cardíaco (Shawn y col., 2007).

La energía consumida durante la $\mathrm{AF}$ es el componente más variable del consumo total de energía y depende de factores como la intensidad y la duración del ejercicio, así como de la composición corporal o la eficacia neta del trabajo. Además, guarda una relación inversa con el nivel de adiposidad (Adams, 2002). Es evidente que la AF es un excelente medio incluso pensando únicamente en gastar energía y evitar acumularla en forma de grasa.

A pesar de que para las personas con desórdenes neurológicos, los beneficios de la práctica regular de $\mathrm{AF}$ son mucho más relevantes que para el resto de la población sigue siendo la porción de la población más sedentaria o inactiva, ya que, además, se enfrentan a problemas adicionales para acceder a la práctica de AF (Rimmer y Rowland, 2008). Si bien diferentes estudios destacan los relevantes beneficios del EF en particular para personas con LM (Dallmeijer y van der Woude, 2001; Dallmeijer, Zentgraaff, Zijp y Woude, 2004; Brizuela, Sinz, Aranda y Martínez, 2010; Figoni, 1993; Haisma y col., 2006; Nash, 2005), son contados los que analizan otras alteraciones que generan mayor impedimento para la práctica de EF, como la PC o la ADF (Haisma y col., 2006).

Existe un acuerdo común sobre la necesidad de la práctica habitual de EF para las personas con estos desórdenes neurológicos y así lo recomienda el Colegio Americano de Medicina del Deporte (Garber y col., 2011). En concreto, se recomienda el entrenamiento de la flexibilidad, la movilidad articular y la fuerza muscular, el entrenamiento de la propiocepción y la estabilidad postural (Schuhfried, Mittermaier, Jovanovic, Pieber y Paternostro-Sluga, 2005), la coordinación muscular, el equilibrio y los ejercicios vestibulares (Ilg y col., 2009).

Además, y de forma especial, se acuerda la conveniencia de realizar ejercicio aeróbico como un modo de mantener el nivel de CF (Dallmeijer y van der Woude, 2001) y mejorar la salud y en definitiva la calidad de vida (Hjeltnes y Wallberg-Henriksson, 1998; Goosey-Tolfrey y Sindall, 2007). El pedaleo de brazos (PB) es un ejercicio aeróbico efectivo para personas que no pueden utilizar los miembros inferiores (Hicks y col., 2003) y se ha demostrado que su práctica regular tiene efectos positivos sobre el sistema cardiorrespiratorio (Valent y col., 2009; Brizuela y col., 2010; Hicks y col., 2010), sobre la resistencia muscular (Valent y col., 2009) y además aumenta la capacidad física y la fuerza muscular (Hicks y col., 2010; Hicks y col., 2003; Brizuela y col., 2010). 
González-Carbonell, I.; Brizuela, G., y Romero-Ávila, J. L. (2016). Pedaleo de brazos en personas con lesión medular, parálisis cerebral o ataxia cerebelosa: Parámetros fisiológicos. RICYDE. Revista internacional de ciencias del deporte. 46(12), 359-381. http://dx.doi.org/10.5232/ricyde2016.04602

Si se quiere planificar o dirigir un programa de entrenamiento para personas con alto grado de afectación neurológica, sea por LM, PC o ataxia, la información aportada sigue siendo sumamente escasa, especialmente para ajustar o dosificar la carga de trabajo. Uno de los problemas principales sería depositar un exceso de confianza en la percepción subjetiva del esfuerzo (PE) de la propia persona, información que llega asociada a la ventilación pulmonar y la actividad muscular, y puede verse afectada por el propio desorden neurológico.

En definitiva, es imprescindible conocer la respuesta fisiológica específica de personas con LM, PC y ADF, con el fin de planificar adecuadamente su AF. Del mismo modo, su GE durante el ejercicio podría ser muy distinto según el tipo de desorden o del grado de afectación, y su organismo tendrá unas demandas energéticas muy distintas en la búsqueda del equilibrio energético saludable. Factores como la intensidad y la duración del ejercicio tienen una especial relevancia para la prevención y tratamiento de cardiopatías, problemas respiratorios, el sobrepeso o la diabetes, que son las principales causas de mortalidad en personas con LM cervical, PC y ADF.

El objetivo del presente estudio es conocer el efecto del ejercicio de pedaleo de brazos sobre la respuesta fisiológica, así como su percepción del esfuerzo, de personas con Lesión Medular, Parálisis Cerebral o Ataxia de Friedreich, comparando su respuesta cardíaca, respiratoria y de gasto energético.

\section{Método}

El presente estudio se desarrolló de forma simultánea a una AF adaptada ofertada a personas con diferentes tipos y grados de discapacidad, de diferentes edades, en el que todos los participantes fueron adquiriendo una notable adherencia al EF y se fueron familiarizando con los diferentes métodos de ejercicio recomendados en la literatura, y de manera especial, con el PB estático en el gimnasio. Todos los participantes fueron seleccionados de este grupo de personas habituadas al EF regular.

\section{Participantes}

Se establecieron cinco grupos de participantes, según el tipo y el nivel de afectación neurológica (Tablas 1 y 2):

Tabla 1. Características de los participantes: LMA: Lesión Medular C5-C6; LMB: Lesión Medular C6-C7; PC: Parálisis Cerebral; ADF: Ataxia de Friedreich; F: Femenino; M: Masculino.

\begin{tabular}{ccccc}
\hline Grupo & Número & Edad & Género & Masa \\
\hline LMA & 4 & $35,7 \pm 10,9$ & $4 \mathrm{M}$ & $75,5 \pm 8,3$ \\
\hline LMB & 4 & $40,7 \pm 6,4$ & $4 \mathrm{M}$ & $74,7 \pm 8,1$ \\
\hline PC & 4 & $40,2 \pm 8,0$ & 1 F - 3 M & $49,2 \pm 10,1$ \\
\hline ADF & 4 & $34,5 \pm 2,5$ & 4 F & $59,7 \pm 13,6$ \\
\hline Control & 16 & $25,8 \pm 6,1$ & 8 F - 8 M & $66,8 \pm 11,4$ \\
\hline Total & $\mathbf{3 2}$ & $\mathbf{3 5 , 4} \pm \mathbf{6 , 8}$ & $\mathbf{1 3}$ F - 19 M & $\mathbf{6 5 , 2} \pm \mathbf{1 0 , 3}$ \\
\hline
\end{tabular}


González-Carbonell, I.; Brizuela, G., y Romero-Ávila, J. L. (2016). Pedaleo de brazos en personas con lesión medular, parálisis cerebral o ataxia cerebelosa: Parámetros fisiológicos. RICYDE. Revista internacional de ciencias del deporte. 46(12), 359-381. http://dx.doi.org/10.5232/ricyde2016.04602

Tabla 2. Clasificación del grado de afectación neurológica de los participantes: ASIA (American Spinal Injury Association); GMFCS (Gross Motor Function Classification System); ICARS (International Cooperative ADF Rating Scale)

\begin{tabular}{|c|c|c|c|}
\hline Participante & Grupo & Valoración & $\begin{array}{c}\text { Tiempo con } \\
\text { afectación }\end{array}$ \\
\hline 1 & \multirow{4}{*}{ LMA } & ASIA C5-C6 B & 10 \\
\hline 2 & & ASIA C5 A & 15 \\
\hline 3 & & ASIA C5 A & 4 \\
\hline 4 & & ASIA C5-C6 C & 1 \\
\hline 5 & \multirow{4}{*}{ LMB } & ASIA C7 A & 5 \\
\hline 6 & & ASIA C6 B & 18 \\
\hline 7 & & ASIA C6-C7 A & 30 \\
\hline 8 & & ASIA C6-C7 A & 11 \\
\hline 9 & \multirow{4}{*}{$\mathrm{PC}$} & GMFCS IV & \multirow{4}{*}{$\begin{array}{c}\text { Desde } \\
\text { nacimiento }\end{array}$} \\
\hline 10 & & GMFCS V & \\
\hline 11 & & GMFCS IV & \\
\hline 12 & & GMFCS IV & \\
\hline 13 & \multirow{4}{*}{$\mathrm{ADF}$} & ICARS: $49 / 100$ & \multirow{4}{*}{$\begin{array}{l}\text { Progresivo } \\
\text { genético }\end{array}$} \\
\hline 14 & & ICARS: $49 / 100$ & \\
\hline 15 & & ICARS: $69 / 100$ & \\
\hline 16 & & ICARS: $75 / 100$ & \\
\hline
\end{tabular}

Los miembros del grupo experimental realizaban habitualmente EF de pedaleo de brazos, $\mathrm{y}$ llevaban realizándolo al menos durante los 6 meses previos al estudio, estando por lo tanto familiarizados con este tipo de ejercicio. Los miembros del grupo Control eran estudiantes universitarios, practicantes habituales de EF y/o Deporte.

A pesar de que el estudio se realizó desarrollando una actividad de pedaleo de brazos con una intensidad de trabajo a la que todos los participantes (excepto los del grupo Control) estaban familiarizados y no se les realizó ninguna medición invasiva, todos los participantes fueron informados del riesgo que implicaba su participación en el estudio, y dieron su consentimiento firmado (o verbal grabado, algunas personas con PC y ADF) para formar parte del mismo. El estudio y sus protocolos fueron desarrollados conforme a los principios éticos de la Universidad de Valencia y el CIOMS (Council for Internacional Organizations of Medical Sciences).

\section{Variables}

Las variables definidas para el estudio fueron las siguientes: FC: Frecuencia cardíaca $\left(\mathrm{min}^{-1}\right)$. FR: Frecuencia respiratoria $\left(\mathrm{min}^{-1}\right)$. VT: Volumen corriente (1). VE: Volumen espirado (1 . $\left.\min ^{-1}\right) . \mathrm{VO}_{2} \mathrm{R}$ : Consumo de Oxígeno relativo $\left(\mathrm{ml} \mathrm{O}_{2} \cdot \min ^{-1} \cdot \mathrm{kg}^{-1}\right) . \mathrm{VO}_{2} / \mathrm{FC}$ : Pulso de Oxígeno $\left(\mathrm{ml} \mathrm{O}_{2}\right)$. VE/ $/ \mathrm{VO}_{2}$ : Equivalente ventilatorio de $\mathrm{O}_{2} . \mathrm{VE} / \mathrm{VCO}_{2}$ : Equivalente ventilatorio de $\mathrm{CO}_{2}$. $\mathrm{R}$ : Cociente respiratorio.

\section{Instrumentos}

Los instrumentos de medición utilizados fueron los siguientes:

- Ergómetro de brazos MONARK (Rehab Trainer 881 E). Permite realizar el pedaleo de brazos ajustando la carga por fricción, con un rango de ajuste entre $0 \mathrm{~W}$ y $100 \mathrm{~W}$, resolución de $5 \mathrm{~W}$ y precisión de ajuste de la carga de $\pm 2,5 \mathrm{~W}$ (Figura 1).

- Analizador de gases portátil Cosmed k4b2 (Cosmed, Roma, Italia): Instrumento para la medición de la respuesta cardiorrespiratoria al ejercicio. Con este sistema se midieron (y calcularon) las variables: $\mathrm{FC}, \mathrm{FR}, \mathrm{VT}, \mathrm{VE}, \mathrm{VO}_{2} \mathrm{R}, \mathrm{VO}_{2} / \mathrm{FC}, \mathrm{VE} / \mathrm{VO}_{2}$, $\mathrm{VE} / \mathrm{VCO}_{2}, \mathrm{R}$ (Figura 1 ). 
González-Carbonell, I.; Brizuela, G., y Romero-Ávila, J. L. (2016). Pedaleo de brazos en personas con lesión medular, parálisis cerebral o ataxia cerebelosa: Parámetros fisiológicos. RICYDE. Revista internacional de ciencias del deporte. 46(12), 359-381. http://dx.doi.org/10.5232/ricyde2016.04602

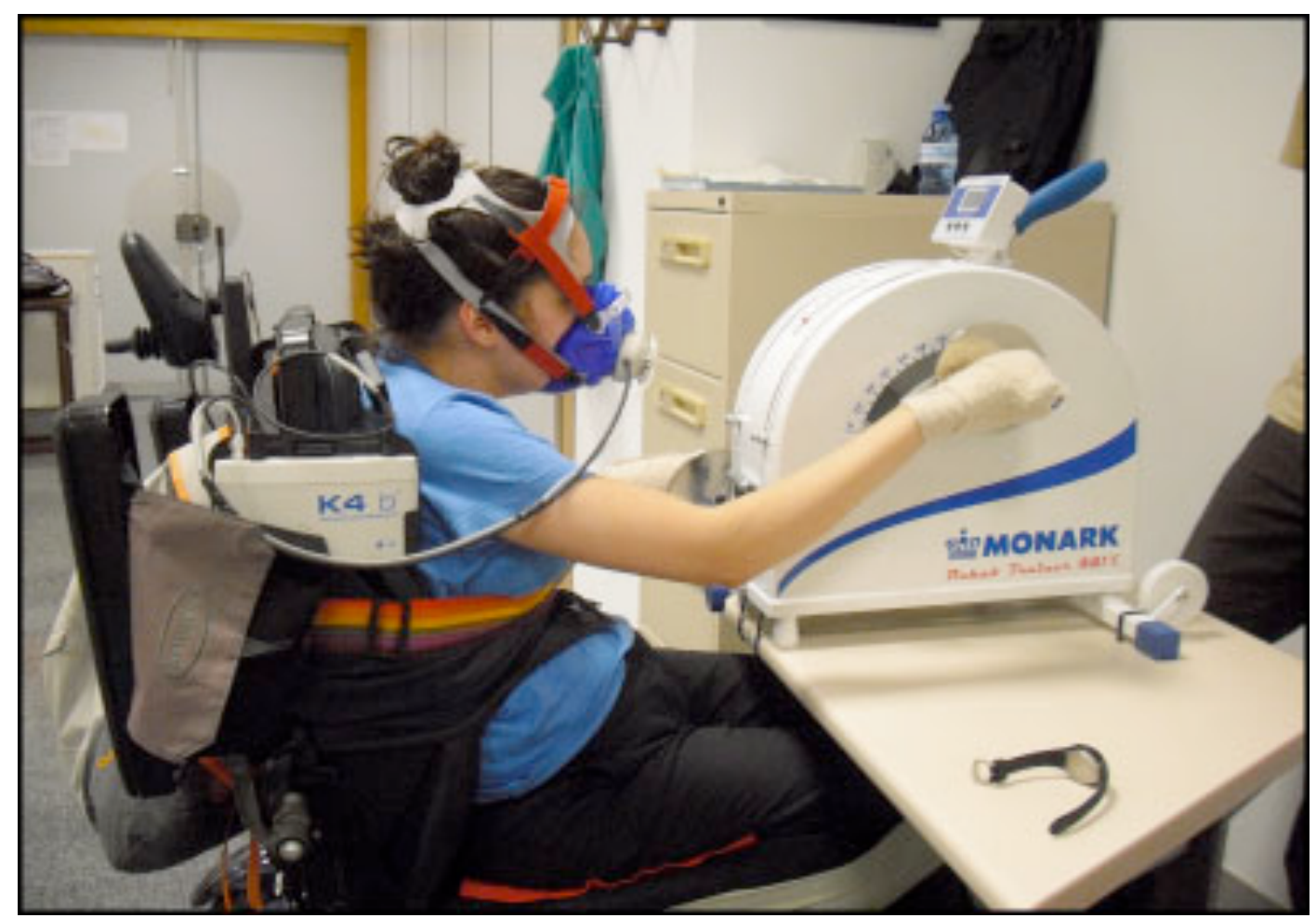

Figura 1. Desarrollo de los experimentos con el Ergómetro de brazos y el analizador de gases.

- Escala modificada de Percepción subjetiva del esfuerzo (Borg, 1982). Mediante esta escala (Tabla 3), cada uno de los participantes valoró la intensidad del esfuerzo que había realizado en cada una de las pruebas, de manera subjetiva y tras la finalización de cada una de ellas.

Tabla 3. Escala modificada de Borg (Borg, 1982), utilizada para la medición de la percepción subjetiva del esfuerzo (PE).

\begin{tabular}{cc}
\hline Valor & Apreciación \\
\hline 0 & Nada \\
\hline 0,5 & Muy muy leve \\
\hline 1 & Muy leve \\
\hline 2 & Leve \\
\hline 3 & Moderada \\
\hline 4 & Algo fuerte \\
\hline 5 & Fuerte o intensa \\
\hline 6 & - \\
\hline 7 & Muy fuerte \\
\hline 8 & - \\
\hline 9 & Muy, muy fuerte (submáxima) \\
\hline 10 & Máxima \\
\hline
\end{tabular}

\section{Procedimientos}

Las mediciones se realizaron en un laboratorio con un ambiente tranquilo (sin otra actividad) donde se controlaron las condiciones de temperatura (entre $20{ }^{\circ} \mathrm{C}$ y $22{ }^{\circ} \mathrm{C}$ ) y de humedad relativa (entre $40 \%$ y $60 \%$ ). Como requisito previo a la prueba se exigió a los participantes no haber realizado esfuerzos intensos desde el día anterior a la prueba, vestir con ropa 
González-Carbonell, I.; Brizuela, G., y Romero-Ávila, J. L. (2016). Pedaleo de brazos en personas con lesión medular, parálisis cerebral o ataxia cerebelosa: Parámetros fisiológicos. RICYDE. Revista internacional de ciencias del deporte. 46(12), 359-381. http://dx.doi.org/10.5232/ricyde2016.04602

deportiva cómoda, no haber ingerido bebidas estimulantes ni alimentos 2 horas previas a la prueba y haber vaciado la vejiga (especialmente a las personas con LM) durante la última hora. Del mismo modo, se fijaron una serie de criterios de interrupción de la prueba que incluían disnea severa, FC muy elevada, malestar del participante, signos de vértigo o mareo. Como medida de seguridad se monitorizó la FC en tiempo real, durante el transcurso de la prueba.

Para asegurarse del correcto funcionamiento y de la validez de los datos obtenidos mediante el analizador de gases se realizó una adecuada calibración previa del instrumento antes de cada sesión de medición. Se definieron las dos intensidades de trabajo en función del peso de cada participante y se cumplimentaron los datos de la hoja de registro. Se procedió a la colocación del cardiotacómetro, de la máscara adecuada para cada persona, se colocó la turbina y se le ajustó mediante el soporte de cabeza.

El protocolo del ejercicio dio comienzo con un calentamiento de entre 6 min y 8 min, un reajuste de la posición del participante en su silla, las sujeciones de las manos a las empuñaduras, de acuerdo a sus propias sensaciones de comodidad y a la impresión de los investigadores. A continuación se realizaron 2 series de $5 \mathrm{~min}$ de pedaleo de brazos continuo con el ergómetro a 2 intensidades diferentes $\left(0,2 \mathrm{~W} \cdot \mathrm{kg}^{-1}\right.$ y $\left.0,4 \mathrm{~W} \cdot \mathrm{kg}^{-1}\right)$, con un descanso de entre $1 \mathrm{~min}$ y $2 \mathrm{~min}$ en medio de ambas series. Todas las pruebas se realizaron a una cadencia de pedaleo de $20 \mathrm{~min}^{-1}$.

Todos los participantes (salvo el grupo Control) realizaron el ejercicio sentados en sus propias sillas de ruedas y contaron con un sistema de estabilización/fijación de su tronco y de apoyo de sus pies, elementos que permitiesen la sujeción pasiva de las manos, por medio de vendajes, cinturones o fajas, según fuera necesario. Los participantes del grupo Control realizaron la prueba montados en la misma silla de ruedas, fijando una distancia que permitiera la extensión casi completa del codo y manteniendo el tronco erecto en todo momento así como los pies apoyados en los reposapiés, con el fin de evitar la implicación de otros grupos musculares.

Una vez que el participante ajustó su cadencia de pedaleo a la cadencia indicada $\left(20 \mathrm{~min}^{-1}\right) \mathrm{y}$ la FC se mostró estable, se dio comienzo a la prueba. Durante todo el desarrollo de la prueba se adquirió con el analizador de gases en modo "respiración a respiración". Tras la finalización de la primera prueba se comprobó si era necesario hacer algún ajuste y se reanudó el pedaleo fijando previamente la segunda intensidad. Al finalizar cada prueba, se pidió a cada participante que valorara el esfuerzo percibido/realizado para así completar la ficha de registro con la puntuación correspondiente a su PE en la escala modificada de Borg (Tabla 3).

\section{Tratamiento estadístico}

En primer lugar, se fijó un nivel de significación Alfa $=0,05$ para todas las pruebas estadísticas y se aplicó una prueba de Kolmogorov-Smirnov para estudiar la normalidad de la distribución de todas las variables cuantitativas, sin detectar datos heterocedásticos. A continuación, se llevó a cabo un análisis de varianza (ANOVA), para cada una de las variables, en función de los factores "Intensidad" y "Grupo", analizando además la interacción entre estos dos factores. Debido a que el factor "Grupo" presenta más de dos niveles (concretamente cinco), se utilizó una prueba LSD post hoc de rango múltiple (MRT) para conocer las posibles diferencias entre los niveles del factor. Todas las pruebas estadísticas se llevaron a cabo con la aplicación StatGraphics (versión 16.1). 
González-Carbonell, I.; Brizuela, G., y Romero-Ávila, J. L. (2016). Pedaleo de brazos en personas con lesión medular, parálisis cerebral o ataxia cerebelosa: Parámetros fisiológicos. RICYDE. Revista internacional de ciencias del deporte. 46(12), 359-381. http://dx.doi.org/10.5232/ricyde2016.04602

\section{Resultados}

La FC mostró (Tabla 4) diferencias estadísticamente significativas $(\mathrm{p}<0,001)$ entre las dos intensidades de ejercicio analizadas para todos los grupos, correspondiendo el mayor valor en todos los casos a la Intensidad 2, a excepción del grupo PC, sin diferencias (post hoc MRT) entre las dos intensidades. Los grupos Control y LMB obtuvieron valores de FC similares entre ellos $(\mathrm{p}<0,001)$ y los más bajos, seguidos del grupo LMA, con valores más altos, seguido del grupo ADF y finalmente del grupo PC, con los valores más altos de todos los grupos. La interacción entre los factores Intensidad y Grupo resultó significativa $(p<0,001)$ para FC.

La FR mostró (Tabla 4) diferencias estadísticamente significativas $(\mathrm{p}<0,001)$ entre los dos niveles del factor Intensidad para todos los grupos correspondiendo el mayor valor siempre a la Intensidad 2, excepto para el grupo Control cuyos valores en la Intensidad 1 fueron más altos que la intensidad 2. El grupo Control obtuvo los valores más bajos de FR, seguido del grupo LMB, posteriormente del grupo ADF y del LMA. Finalmente el grupo PC alcanzó los valores más altos de FR. La interacción entre los factores Intensidad y Grupo resultó significativa $(\mathrm{p}<0,001)$ para FR.

El VT mostró (Tabla 4) diferencias estadísticamente significativas $(\mathrm{p}<0,001)$ entre las dos intensidades de ejercicio para todos los grupos, excepto para el grupo PC, que no mostró diferencias significativas (post hoc MRT) en VT entre ambos niveles del factor Intensidad. El grupo PC obtuvo los valores más bajos de VT, seguido del grupo ADF, del grupo LMA, del grupo LMB y por último, del grupo Control. La interacción entre los factores Intensidad y Grupo resultó significativa $(\mathrm{p}<0,001)$ para VT.

La VE (Tabla 5) mostró diferencias estadísticamente significativas $(\mathrm{p}<0,001)$ entre los dos niveles del factor Intensidad para todos los grupos, correspondiendo el valor más alto siempre a la Intensidad 2, excepto para el grupo PC, sin diferencias significativas (post hoc MRT). El grupo Control obtuvo los valores más bajos de VE, seguido del grupo LMB, del grupo ADF, del grupo LMA y finalmente por el grupo PC. La interacción entre los factores Intensidad y Grupo resultó significativa $(\mathrm{p}<0,001)$ para VE.

El $\mathrm{VO}_{2} \mathrm{R}$ (Tabla 5) mostró diferencias estadísticamente significativas $(\mathrm{p}<0,001)$ entre los dos niveles del factor Intensidad para todos los grupos, correspondiendo el valor más alto siempre a la Intensidad 2. Los grupos LMA y LMB obtuvieron los valores más bajos de $\mathrm{VO}_{2} \mathrm{R}$, seguido del grupo Control, del grupo ADF y del grupo PC. La interacción entre los factores Intensidad y Grupo no resultó significativa $(\mathrm{p}>0,05)$ para $\mathrm{VO}_{2} \mathrm{R}$.

$\mathrm{El} \mathrm{VO}_{2} / \mathrm{FC}($ Tabla 5) mostró diferencias estadísticamente significativas $(\mathrm{p}<0,001)$ entre los dos niveles del factor Intensidad para todos los grupos, correspondiendo el valor más alto siempre a la Intensidad 2, excepto para el grupo LMA que no mostró diferencias significativas (post hoc MRT) en $\mathrm{VO}_{2} / \mathrm{FC}$, entre ambos niveles del factor Intensidad. El grupo $\mathrm{ADF}$ obtuvo los valores más bajos de $\mathrm{VO}_{2} / \mathrm{FC}$, seguido del grupo LMA, del grupo PC y de los grupos LMB y Control, sin diferencias significativas entre ellos (post hoc MRT). La interacción entre los factores Intensidad y Grupo resultó significativa $(\mathrm{p}<0,001)$ para $\mathrm{VO}_{2} / \mathrm{FC}$. 
González-Carbonell, I.; Brizuela, G., y Romero-Ávila, J. L. (2016). Pedaleo de brazos en personas con lesión medular, parálisis cerebral o ataxia cerebelosa: Parámetros fisiológicos. RICYDE. Revista internacional de ciencias del deporte. 46(12), 359-381. http://dx.doi.org/10.5232/ricyde2016.04602

El VE/ $/ \mathrm{VO}_{2}$ (Tabla 6) mostró diferencias estadísticamente significativas (post hoc MRT) entre los dos niveles del factor Intensidad para, para el grupo LMA y para el grupo ADF, correspondiendo el valor más alto a la Intensidad 2. También se encontraron diferencias estadísticamente significativas (post hoc MRT) entre los dos niveles del factor Intensidad para los grupos Control, LMB y PC, pero en estos casos, el valor más alto correspondió a la Intensidad 1. Para el grupo LMB no se encontraron diferencias significativas (post hoc MRT) en $\mathrm{VE} / \mathrm{VO}_{2}$ entre ambos niveles del factor Intensidad. El grupo Control obtuvo los valores más bajos de $\mathrm{VE} / \mathrm{VO}_{2}$, seguido del grupo $\mathrm{LMB}$, del grupo $\mathrm{ADF}$, del grupo LMA y del grupo PC. La interacción entre los factores Intensidad y Grupo resultó significativa $(p<0,001)$ para $\mathrm{VE} / \mathrm{VO}_{2}$.

$\mathrm{El} \mathrm{VE} / \mathrm{VCO}_{2}$ (Tabla 6) mostró diferencias estadísticamente significativas $(\mathrm{p}=0,0262)$ entre los dos niveles del factor Intensidad únicamente para el grupo Control, correspondiendo el valor más alto a la Intensidad 1. El grupo Control obtuvo los valores más bajos de $\mathrm{VE} / \mathrm{VCO}_{2}$, seguido del grupo ADF, de los grupos LMA y LMB (sin diferencias significativas entre ambos grupos) y del grupo PC. La interacción entre los factores Intensidad y Grupo resultó significativa $(\mathrm{p}=0,0005)$ para $\mathrm{VE} / \mathrm{VCO}_{2}$.

El R (Tabla 6) mostró diferencias estadísticamente significativas $(\mathrm{p}<0,001)$ entre los dos niveles del factor Intensidad para los grupos LMA, LMB, PC y ADF, correspondiendo el valor más alto a la Intensidad 2, a excepción del grupo PC con el valor más alto a Intensidad 1. El grupo Control no mostró diferencias significativas (post hoc MRT) en R entre ambos niveles del factor Intensidad. El grupo Control obtuvo los valores más bajos de R, seguido del grupo LMB, del grupo ADF y de los grupos PC y LMA (sin diferencias significativas entre estos grupos). La interacción entre los factores Intensidad y Grupo resultó significativa ( $\mathrm{p}<$ $0,001)$ para $\mathrm{R}$.

La PE (Tabla 7) mostró diferencias estadísticamente significativas $(\mathrm{p}<0,001)$ entre los niveles del factor Intensidad para todos los grupos, siendo en todos los casos la PE más alta a Intensidad 2. También se encontraron diferencias estadísticamente significativas $(p<0,001)$ entre los niveles del factor Grupo, el grupo Control mostró los valores más bajos, seguido del grupo LMB, del grupo ADF, del grupo PC y finalmente por el grupo LMA. La interacción entre los factores Intensidad y Grupo resultó significativa $(p<0,001)$ para PE. 
González-Carbonell, I.; Brizuela, G., y Romero-Ávila, J. L. (2016). Pedaleo de brazos en personas con lesión medular, parálisis cerebral o ataxia cerebelosa: Parámetros fisiológicos. RICYDE. Revista internacional de ciencias del deporte. 46(12), 359-381. http://dx.doi.org/10.5232/ricyde2016.04602

Tabla 4: Resultados del ANOVA según los factores Grupo e Intensidad, para las variables Frecuencia Cardíaca (FC), Frecuencia Respiratoria (FR) y Volumen corriente (VT).

\begin{tabular}{|c|c|c|c|c|c|c|c|c|c|c|c|c|}
\hline \multirow{3}{*}{ Variable } & \multirow{3}{*}{ Unid } & \multirow{3}{*}{ Fac Intensidad } & \multicolumn{10}{|c|}{ Factor Grupo } \\
\hline & & & \multicolumn{2}{|c|}{ Control } & \multicolumn{2}{|c|}{ LMA } & \multicolumn{2}{|c|}{ LMB } & \multicolumn{2}{|c|}{$\mathrm{PC}$} & \multicolumn{2}{|c|}{$\mathrm{ADF}$} \\
\hline & & & Media \pm SE & MRT & Media \pm SE & MRT & $\operatorname{Media} \pm \mathrm{SE}$ & MRT & $\operatorname{Media} \pm \mathrm{SE}$ & MRT & Media $\pm \mathrm{SE}$ & MRT \\
\hline \multirow{6}{*}{ FC } & \multirow{2}{*}{$\min ^{-1}$} & I1 (0,2 W/Kg) & $77,06 \pm 0,46$ & \multirow{2}{*}{$\mathrm{I} 1<\mathrm{I} 2$} & $88,93 \pm 0,90$ & \multirow{2}{*}{$\mathrm{I} 1<\mathrm{I} 2$} & $76,55 \pm 0,92$ & \multirow{2}{*}{$\mathrm{I} 1<\mathrm{I} 2$} & $143,14 \pm 0,59$ & \multirow{2}{*}{$\mathrm{I} 1=\mathrm{I} 2$} & $118,81 \pm 0,82$ & \multirow{2}{*}{$\mathrm{I} 1<\mathrm{I} 2$} \\
\hline & & I2 $(0,4 \mathrm{~W} / \mathrm{Kg})$ & $83,04 \pm 0,47$ & & $106,52 \pm 0,96$ & & $89,17 \pm 0,89$ & & $143,21 \pm 0,58$ & & $130,01 \pm 0,84$ & \\
\hline & \multicolumn{2}{|c|}{ MRT post hoc } & \multicolumn{10}{|c|}{$0=2<1<4<3$} \\
\hline & \multicolumn{2}{|c|}{ Factor grupo } & \multicolumn{10}{|c|}{$\mathrm{p}<0,0001$} \\
\hline & \multicolumn{2}{|c|}{ Factor intensidad } & \multicolumn{10}{|c|}{$\mathrm{p}<0,0001$} \\
\hline & \multicolumn{2}{|c|}{ Interacción Factores } & \multicolumn{10}{|c|}{$\mathrm{p}<0,0001$} \\
\hline \multirow{6}{*}{ FR } & \multirow{2}{*}{$\min ^{-1}$} & $\mathrm{I} 1(0,2 \mathrm{~W} / \mathrm{Kg})$ & $18,91 \pm 0,39$ & \multirow{2}{*}{$\mathrm{I} 1>\mathrm{I} 2$} & $24 \pm 0,69$ & \multirow{2}{*}{$\mathrm{I} 1<\mathrm{I} 2$} & $20,37 \pm 0,77$ & \multirow{2}{*}{$\mathrm{I} 1<\mathrm{I} 2$} & $53,47 \pm 0,49$ & \multirow{2}{*}{$\mathrm{I} 1<\mathrm{I} 2$} & $23,48 \pm 0,69$ & \multirow[b]{2}{*}{ I1 $<$ I2 } \\
\hline & & $\mathrm{I} 2(0,4 \mathrm{~W} / \mathrm{Kg})$ & $18,33 \pm 0,40$ & & $29,94 \pm 0,75$ & & $23,71 \pm 0,69$ & & $55,97 \pm 0,48$ & & $25,39 \pm 0,71$ & \\
\hline & \multicolumn{2}{|c|}{ MRT post hoc } & & & & & $0<2$ & $<1<3$ & & & & \\
\hline & Factor G & rupo & & & & & & 0001 & & & & \\
\hline & Factor I & tensidad & & & & & & 0001 & & & & \\
\hline & Interacc & ión Factores & & & & & & 0001 & & & & \\
\hline & , & I1 $(0,2 \mathrm{~W} / \mathrm{Kg})$ & $0,77 \pm 0,009$ & & $0,73 \pm 0,02$ & & $0,74 \pm 0,02$ & & $0,68 \pm 0,01$ & & $0,66 \pm 0,02$ & \\
\hline & & $\mathrm{I} 2(0,4 \mathrm{~W} / \mathrm{Kg})$ & $0,93 \pm 0,009$ & $\mathrm{I} 1<\mathrm{I} 2$ & $0,78 \pm 0,02$ & $\mathrm{I} 1<\mathrm{I} 2$ & $0,87 \pm 0,02$ & I2 & $0,66 \pm 0,01$ & $\mathrm{I} 1=\mathrm{I} 2$ & $0,77 \pm 0,02$ & $\mathrm{I} 1<\mathrm{I} 2$ \\
\hline $\mathrm{VT}$ & MRT po & st hoc & & & & & $3<4$ & $<2<0$ & & & & \\
\hline & Factor G & rupo & & & & & & 0001 & & & & \\
\hline & Factor I & tensidad & & & & & & 0001 & & & & \\
\hline & Interacc & ión Factores & & & & & & 0001 & & & & \\
\hline
\end{tabular}


González-Carbonell, I.; Brizuela, G., y Romero-Ávila, J. L. (2016). Pedaleo de brazos en personas con lesión medular, parálisis cerebral o ataxia cerebelosa: Parámetros fisiológicos. RICYDE. Revista internacional de ciencias del deporte. 46(12), 359-381. http://dx.doi.org/10.5232/ricyde2016.04602

Tabla 5: Resultados del ANOVA según los factores Grupo e Intensidad, para las variables Volumen Espirado (VE), Consumo de Oxígeno Relativo (VO 2 R) y Pulso de Oxígeno $\left(\mathrm{VO}_{2} / \mathrm{FC}\right)$.

\begin{tabular}{|c|c|c|c|c|c|c|c|c|c|c|c|c|}
\hline \multirow{3}{*}{ Variable } & \multirow{3}{*}{ Unid } & \multirow{3}{*}{ Fac Intensidad } & \multicolumn{10}{|c|}{ Factor Grupo } \\
\hline & & & \multicolumn{2}{|c|}{ Control } & \multicolumn{2}{|c|}{ LMA } & \multicolumn{2}{|c|}{ LMB } & \multicolumn{2}{|c|}{$\mathrm{PC}$} & \multicolumn{2}{|c|}{ ADF } \\
\hline & & & Media $\pm \mathrm{SE}$ & MRT & $\operatorname{Media} \pm \mathrm{SE}$ & MRT & $\operatorname{Media} \pm \mathrm{SE}$ & MRT & Media $\pm \mathrm{SE}$ & MRT & $\operatorname{Media} \pm \mathrm{SE}$ & MRT \\
\hline \multirow{6}{*}{ VE } & \multirow{2}{*}{$1 \cdot \min ^{-1}$} & I1 $(0,2 \mathrm{~W} / \mathrm{Kg})$ & $13,55 \pm 0,31$ & \multirow{2}{*}{$\mathrm{I} 1<\mathrm{I} 2$} & $16,47 \pm 0,55$ & \multirow{2}{*}{$\mathrm{I} 1<\mathrm{I} 2$} & $13,27 \pm 0,61$ & \multirow{2}{*}{$\mathrm{I} 1<\mathrm{I} 2$} & $36,97 \pm 0,39$ & \multirow[b]{2}{*}{$\mathrm{I} 1=\mathrm{I} 2$} & $15,48 \pm 0,56$ & \multirow{2}{*}{$\mathrm{I} 1<\mathrm{I} 2$} \\
\hline & & $\mathrm{I} 2(0,4 \mathrm{~W} / \mathrm{Kg})$ & $16,02 \pm 0,32$ & & $21,27 \pm 0,60$ & & $18,22 \pm 0,55$ & & $37,08 \pm 0,39$ & & $19,52 \pm 0,57$ & \\
\hline & \multicolumn{2}{|c|}{ MRT post hoc } & \multicolumn{10}{|c|}{$0<2<4<1<3$} \\
\hline & \multicolumn{2}{|c|}{ Factor grupo } & \multicolumn{10}{|c|}{$\mathrm{p}<0,0001$} \\
\hline & \multicolumn{2}{|c|}{ Factor intensidad } & \multicolumn{10}{|c|}{$\mathrm{p}<0,0001$} \\
\hline & \multicolumn{2}{|c|}{ Interacción Factores } & \multicolumn{10}{|c|}{$p<0,0001$} \\
\hline \multirow{6}{*}{$\mathrm{VO}_{2} \mathrm{R}$} & $\mathrm{ml} \mathrm{O}$. & $\mathrm{I} 1(0,2 \mathrm{~W} / \mathrm{Kg})$ & $6,82 \pm 0,11$ & \multirow{2}{*}{$\mathrm{I} 1<\mathrm{I} 2$} & $5,99 \pm 0,19$ & \multirow{2}{*}{$\mathrm{I} 1<\mathrm{I} 2$} & $5,69 \pm 0,21$ & \multirow{2}{*}{$\mathrm{I} 1<\mathrm{I} 2$} & $13,84 \pm 0,14$ & & $7,79 \pm 0,19$ & \multirow{2}{*}{ I1 $<$ I 2} \\
\hline & $\mathrm{Kg}^{-1}$ & $\mathrm{I} 2(0,4 \mathrm{~W} / \mathrm{Kg})$ & $8,53 \pm 0,11$ & & $7,36 \pm 0,21$ & & $7,66 \pm 0,19$ & & $15,14 \pm 0,14$ & I1 $<$ I 2 & $8,96 \pm 0,20$ & \\
\hline & \multicolumn{2}{|c|}{ MRT post hoc } & \multicolumn{10}{|c|}{$2=1<0<4<3$} \\
\hline & \multicolumn{2}{|c|}{ Factor Grupo } & & & & & $\mathrm{p}<0$ & & & & & \\
\hline & Factor In & tensidad & & & & & $\mathrm{p}<0$ & & & & & \\
\hline & Interaccic & on Factores & & & & & $p=0$ & & & & & \\
\hline & $\mathrm{ml} \mathrm{O}_{2}$ & I1 $(0,2 \mathrm{~W} / \mathrm{Kg})$ & $5,84 \pm 0,05$ & & $4,97 \pm 0,10$ & & $5,68 \pm 0,10$ & & $5,09 \pm 0,07$ & & $3,71 \pm 0,97$ & \\
\hline & & $\mathrm{I} 2(0,4 \mathrm{~W} / \mathrm{Kg})$ & $6,69 \pm 0,06$ & I1 $<$ I2 & $5,08 \pm 0,11$ & $\mathrm{I} 1=\mathrm{I} 2$ & $6,69 \pm 0,10$ & I1 $<$ I 2 & $5,50 \pm 0,07$ & I1 $<$ I 2 & $3,99 \pm 0,10$ & $\mathrm{I} 1<\mathrm{I} 2$ \\
\hline $\mathrm{VO}, / \mathrm{FC}$ & MRT pos & thoc & & & & & $4<1<$ & $2=0$ & & & & \\
\hline & Factor $\mathrm{G}_{\mathrm{l}}$ & rupo & & & & & $p<0$ & & & & & \\
\hline & Factor In & tensidad & & & & & $\mathrm{p}<0$ & & & & & \\
\hline & Interaccic & ón Factores & & & & & $\mathrm{p}<0$ & & & & & \\
\hline
\end{tabular}


González-Carbonell, I.; Brizuela, G., y Romero-Ávila, J. L. (2016). Pedaleo de brazos en personas con lesión medular, parálisis cerebral o ataxia cerebelosa: Parámetros fisiológicos. RICYDE. Revista internacional de ciencias del deporte. 46(12), 359-381. http://dx.doi.org/10.5232/ricyde2016.04602

Tabla 6: Resultados del ANOVA según los factores Grupo e Intensidad, para las variables Equivalente Respiratorio de Oxígeno (VE/VO 2 ), Equivalente Respiratorio de Dióxido de Carbono (VE/ $\mathrm{VCO}_{2}$ ) y Cociente respiratorio (R).

\begin{tabular}{|c|c|c|c|c|c|c|c|c|c|c|c|c|}
\hline \multirow{3}{*}{ Variable } & \multirow{3}{*}{ Unid } & \multirow{3}{*}{ Fac Intensidad } & \multicolumn{10}{|c|}{ Factor Grupo } \\
\hline & & & \multicolumn{2}{|c|}{ Control } & \multicolumn{2}{|c|}{ LMA } & \multicolumn{2}{|c|}{ LMB } & \multicolumn{2}{|c|}{$\mathrm{PC}$} & \multicolumn{2}{|c|}{ ADF } \\
\hline & & & $\operatorname{Media} \pm \mathrm{SE}$ & MRT & Media \pm SE & MRT & $\operatorname{Media} \pm \mathrm{SE}$ & MRT & Media $\pm \mathrm{SE}$ & MRT & Media $\pm \mathrm{SE}$ & MRT \\
\hline \multirow{6}{*}{$\mathrm{VE} / \mathrm{VO}_{2}$} & & I1 (0,2 W/Kg) & $30,72 \pm 0,61$ & \multirow{2}{*}{$\mathrm{I} 1>\mathrm{I} 2$} & $40,08 \pm 1,09$ & \multirow{2}{*}{$\mathrm{I} 1<\mathrm{I} 2$} & $35,71 \pm 1,22$ & \multirow{2}{*}{$\mathrm{I} 1=\mathrm{I} 2$} & $53,74 \pm 0,79$ & \multirow{2}{*}{$\mathrm{I} 1>\mathrm{I} 2$} & $36,07 \pm 1,09$ & \multirow{2}{*}{$\mathrm{I} 1<\mathrm{I} 2$} \\
\hline & & $\mathrm{I} 2(0,4 \mathrm{~W} / \mathrm{Kg})$ & $28,88 \pm 0,63$ & & $45,62 \pm 1,19$ & & $34,12 \pm 1,09$ & & $49,50 \pm 0,77$ & & $39,49 \pm 1,12$ & \\
\hline & \multicolumn{2}{|c|}{ MRT post hoc } & \multicolumn{10}{|c|}{$0<2<4<1<3$} \\
\hline & \multicolumn{2}{|c|}{ Factor grupo } & \multicolumn{10}{|c|}{$\mathrm{p}<0,0001$} \\
\hline & \multicolumn{2}{|c|}{ Factor intensidad } & \multicolumn{10}{|c|}{$\mathrm{p}=0,6805$} \\
\hline & \multicolumn{2}{|c|}{ Interacción Factores } & \multicolumn{10}{|c|}{$\mathrm{p}<0,0001$} \\
\hline \multirow{6}{*}{$\mathrm{VE} / \mathrm{VCO}_{2}$} & & $\mathrm{I} 1(0,2 \mathrm{~W} / \mathrm{Kg})$ & $36,97 \pm 0,95$ & \multirow{2}{*}{$\mathrm{I} 1>\mathrm{I} 2$} & $46,30 \pm 1,69$ & \multirow{2}{*}{$\mathrm{I} 1>\mathrm{I} 2$} & $53,82 \pm 1,88$ & \multirow{2}{*}{$\mathrm{I} 1=\mathrm{I} 2$} & $54,93 \pm 1,22$ & \multirow{2}{*}{$\mathrm{I} 1=\mathrm{I} 2$} & $42,37 \pm 1,69$ & \multirow{2}{*}{$\mathrm{I} 1=\mathrm{I} 2$} \\
\hline & & $\mathrm{I} 2(0,4 \mathrm{~W} / \mathrm{Kg})$ & $34,92 \pm 0,98$ & & $48,75 \pm 1,84$ & & $42,19 \pm 1,69$ & & $54,25 \pm 1,19$ & & $43,54 \pm 1,74$ & \\
\hline & \multicolumn{6}{|c|}{ MRT post hoc } & \multicolumn{2}{|c|}{$0<4<1=2<3$} & & & & \\
\hline & \multicolumn{2}{|c|}{ Factor Grupo } & & & & & $\mathrm{p}<$ & 001 & & & & \\
\hline & Factor & Intensidad & & & & & $\mathrm{p}=$ & 262 & & & & \\
\hline & Intera & ción Factores & & & & & $p=$ & 005 & & & & \\
\hline & & $\mathrm{I} 1(0,2 \mathrm{~W} / \mathrm{Kg})$ & 0,83 & $\mathrm{I} 1=\mathrm{I}$ & 0,89 & $\mathrm{I} 1<\mathrm{I} 2$ & 0,81 & $\mathrm{I} 1<\mathrm{I}$ & 0,99 & I2 & 0,85 & \\
\hline & & $\mathrm{I} 2(0,4 \mathrm{~W} / \mathrm{Kg})$ & 0,83 & & 0,97 & & 0,86 & & 0,87 & $11+12$ & 0,90 & $11<12$ \\
\hline $\mathbf{R}$ & $\mathrm{MRT}_{1}$ & ost hoc & & & & & $0<2$ & $<3=1$ & & & & \\
\hline & Factor & Grupo & & & & & $\mathrm{p}<$ & 001 & & & & \\
\hline & Factor & Intensidad & & & & & $\mathrm{p}<$ & 001 & & & & \\
\hline & Intera & ción Factores & & & & & $\mathrm{p}<$ & 001 & & & & \\
\hline
\end{tabular}


González-Carbonell, I.; Brizuela, G., y Romero-Ávila, J. L. (2016). Pedaleo de brazos en personas con lesión medular, parálisis cerebral o ataxia cerebelosa: Parámetros fisiológicos. RICYDE. Revista internacional de ciencias del deporte. 46(12), 359-381. http://dx.doi.org/10.5232/ricyde2016.04602

Tabla 7. Resultados del ANOVA para la variable Percepción del Esfuerzo (PE) según los factores Grupo e Intensidad y su interacción (SE: Error estándar).

\begin{tabular}{cc}
\hline Factor & PE \\
\hline \multicolumn{1}{c}{ Media $\pm \boldsymbol{S E}$} \\
\hline $\begin{array}{c}\mathrm{p}<0,0001) \\
\text { Control }\end{array}$ & $2,11 \pm 0,02$ \\
\hline LMA & $5,93 \pm 0,03$ \\
\hline LMB & $2,81 \pm 0,03$ \\
\hline PC & $3,47 \pm 0,02$ \\
\hline ADF & $2,97 \pm 0,03$ \\
\hline Intensidad \\
$(\mathrm{p}<0,0001)$ \\
\hline 1
\end{tabular}

\begin{tabular}{|c|c|c|}
\hline Grupo & Intensidad & PE \\
\hline \multicolumn{2}{|c|}{$\begin{array}{l}\text { Interacción } \\
(p<0,0001)\end{array}$} & Media $\pm S E$ \\
\hline \multirow{2}{*}{ Control } & 1 & $1,27 \pm 0,03$ \\
\hline & 2 & $2,95 \pm 0,03$ \\
\hline \multirow{2}{*}{ LMA } & 1 & $2,71 \pm 0,04$ \\
\hline & 2 & $9,16 \pm 0,05$ \\
\hline \multirow{2}{*}{ LMB } & 1 & $1,99 \pm 0,05$ \\
\hline & 2 & $3,63 \pm 0,05$ \\
\hline \multirow{2}{*}{$\mathrm{PC}$} & 1 & $2,80 \pm 0,03$ \\
\hline & 2 & $4,14 \pm 0,03$ \\
\hline \multirow{2}{*}{$\mathrm{ADF}$} & 1 & $2,39 \pm 0,05$ \\
\hline & 2 & $3,54 \pm 0,05$ \\
\hline
\end{tabular}

\section{Discusión}

La intensidad del ejercicio realizado no supuso un estrés relevante en el organismo de los participantes del grupo Control y esto se reflejó en los niveles más bajos de FC, VE, FR y $\mathrm{VE} / \mathrm{VO}_{2}$, debido esencialmente a la baja (para este grupo) intensidad del ejercicio. El incremento en la intensidad del ejercicio, supuso un incremento únicamente de la VT, sin variar la FR, lo que refleja, del mismo modo, un incremento muy moderado de la intensidad para este grupo. Por el contrario, el grupo Control (junto al grupo LMB), alcanzó los valores más altos de $\mathrm{VO}_{2} / \mathrm{FC}$, reflejando el grado más alto de eficiencia cardiovascular.

Respecto a la percepción subjetiva del esfuerzo realizado (PE) el grupo Control percibió un incremento del esfuerzo muy próximo a la realidad (131\%) al aumentar la intensidad del ejercicio un $100 \%$.

El grupo LMA es el que se mostró más próximo al límite de sus capacidades respiratorias, cardiovasculares y muy posiblemente musculares, especialmente a la intensidad más alta de ejercicio. Esto se reflejó en sus valores más altos de FR y VE (junto al grupo PC) y los valores máximos de PE. Esta aproximación a su límite de intensidad de ejercicio se reflejó en balanceos exagerados de la cabeza (que arrastraban al tronco), intentando generar más potencia durante el PB. A pesar de que todos los grupos realizaron ejercicio a la misma intensidad (relativa a su peso), que supuso una intensidad ligera o moderada para todos los grupos, la intensidad 2 resultó submáxima o máxima para el grupo LMA. Estos resultados concuerdan con los de Hicks y col., (2003), Hjeltnes (1986) y West, Mills y Krassioukov, (2012), en los que personas con tetraplejia por LM, obtienen menor rendimiento y una capacidad cardiorrespiratoria limitada, en pruebas de esfuerzo máximas, cuando se las compara con personas con LM a inferior nivel. 
González-Carbonell, I.; Brizuela, G., y Romero-Ávila, J. L. (2016). Pedaleo de brazos en personas con lesión medular, parálisis cerebral o ataxia cerebelosa: Parámetros fisiológicos. RICYDE. Revista internacional de ciencias del deporte. 46(12), 359-381. http://dx.doi.org/10.5232/ricyde2016.04602

$\mathrm{El} \mathrm{VO}_{2} / \mathrm{FC}$ también resultó el más bajo para el grupo LMA, y a diferencia del resto de grupos, no se alteró entre ambas intensidades de ejercicio. Esto está claramente relacionado con la interrupción del Sistema Nervioso Simpático (debido a la propia LM), encargado de la estimulación nerviosa del corazón, destacando un volumen sistólico disminuido en personas con tetraplejia (Nash y col., 1991). Si se añade la hipotensión y la dificultad del retorno venoso (también presente en personas con LM alta), se podría explicar el bajo grado de eficiencia del sistema circulatorio y el transporte de oxígeno, destacando que su capacidad de incrementar el $\mathrm{VO}_{2} \mathrm{R}$ está más afectada incluso que la de aumentar su $\mathrm{FC}$, debido probablemente a un déficit básicamente circulatorio.

Los valores medidos de $\mathrm{R}$ aseguran que el grupo LMA (al igual que el grupo PC) estaba mucho más cerca de la utilización del sistema anaeróbico de aporte de energía, esto se reflejó en un aumento fundamentalmente de VE y FR. Este grupo, seguramente habrá estado trabajando a intensidades en las que el componente anaeróbico era muy considerable, acercándose a su límite muscular y, especialmente a Intensidad 2, tenían comprometida la capacidad máxima de su sistema cardiorrespiratorio.

El grupo LMB (junto al grupo Control) obtuvo los valores más bajos de FC y de forma global, ambos grupos con LM mostraron el menor GE $\left(\mathrm{FC} \mathrm{y} \mathrm{VO}_{2} \mathrm{R}\right)$ durante el ejercicio de $\mathrm{PB}$. Esto podría reflejar una mayor eficiencia, quizás debido a estar más familiarizados con el ejercicio realizado, aunque seguramente se deba simplemente a la implicación de menor cantidad de masa muscular, no pudiendo utilizar la musculatura de manos y muñecas, los extensores de los codos y sobre todo musculatura estabilizadora del raquis, a diferencia del resto de grupos. Estos resultados coinciden con estudios donde se comparó el GE durante la realización de distintas actividades, entre personas con y sin LM (Dearwater y col., 1985; Conger y Bassett, 2011) y con otros estudios que comparan un grupo con tetraplejia, frente a otros con paraplejia o sin LM (Monroe y col., 1998; Pelletier, Jones, Latimer-Cheung, Warburton y Hicks, 2013; Buchholz y Pencharz, 2004; Yamasaki y col., 1992). En todos los casos, los grupos con tetraplegia obtuvieron un menor gasto energético.

Hjeltnes (1986) en un estudio hecho a personas con tetraplejia, concluye que el aumento necesario del VE se consigue básicamente aumentando la FR, lo cual coincide plenamente con los resultados del grupo LMA de nuestro estudio. Esto es debido a que las personas con LMA realizan una respiración diafragmática y compensan el menor llenado de los pulmones con una mayor FR. Sin embargo, a partir de los resultados de nuestro estudio, podemos añadir que cuando la LM es algo más baja y compromete menos al sistema cardiorrespiratorio (grupo LMB), los valores de FR aumentan pero es más destacable el incremento de VT, evidentemente debido a la posibilidad de implicar mayor masa de musculatura respiratoria.

El grupo LMB (junto al grupo Control) resultó el más eficiente a nivel cardiovascular con los valores más altos de $\mathrm{VO}_{2} / \mathrm{FC}$, coincidiendo con los valores de $\mathrm{FC}$ donde ambos grupos obtuvieron los valores más bajos. Este hecho permitiría concluir que la eficacia en el transporte y/o absorción del oxígeno no se ve influenciada por la interrupción parcial del sistema simpático que actúa en la activación cardíaca en el grupo de LMB, a las intensidades analizadas en el presente estudio, a pesar de que su nivel de lesión (C6-C7) está muy próximo al de LMA (C5-C6).

El grupo LMB percibió (PE) un incremento del esfuerzo del $83 \%$, muy próximo a la realidad (100\% mayor a Intensidad 2). Sin embargo el grupo LMA, acercándose mucho más a sus capacidades máximas de esfuerzo y posiblemente sobrepasando su umbral anaeróbico, percibió un incremento del $238 \%$ para el mismo incremento real. 
González-Carbonell, I.; Brizuela, G., y Romero-Ávila, J. L. (2016). Pedaleo de brazos en personas con lesión medular, parálisis cerebral o ataxia cerebelosa: Parámetros fisiológicos. RICYDE. Revista internacional de ciencias del deporte. 46(12), 359-381. http://dx.doi.org/10.5232/ricyde2016.04602

A modo de recomendación, las personas con tetraplejia por LM cervical completa no alcanzan a generar niveles de estrés elevados para su sistema cardiorrespiratorio durante el pedaleo de brazos estático, debido fundamentalmente a la baja cantidad de masa muscular que pueden activar voluntariamente. Esto supone un muy bajo nivel de riesgo de sobrepasar los límites de intensidad recomendables para un ejercicio saludable desde punto de vista cardiovascular, aunque podrían estar alcanzando su límite de tolerancia o resistencia muscular al ejercicio (importante para el entrenamiento muscular). Debido a esto, no parece necesaria la monitorización constante de la FC con el fin de evitar sobrepasar los límites saludables, aunque sí podría ser útil para controlar diferentes zonas de intensidad de entrenamiento.

Finalmente, habiendo comprobado que el GE de las personas con LM cervical durante el pedaleo de brazos es similar o inferior al del grupo Control y, de acuerdo a la gran cantidad de estudios que confirman que su consumo calórico diario es muy inferior al de una persona de su mismo peso, parece razonable recomendar el control a la baja de la ingesta calórica (a ajustar por un profesional) así como la realización de ejercicio físico regular que propiciará un mayor gasto energético, la reducción de la masa grasa, el aumento de masa muscular y una mayor estimulación y adaptación positiva del sistema cardiorrespiratorio.

El grupo ADF mostró un estrés mayor durante el ejercicio con una FC $55 \%$ más elevada (frente a los grupos Control y LMB) y los valores más altos de $\mathrm{VO}_{2} \mathrm{R}$ (junto al grupo $\mathrm{PC}$ ), requiriendo una mayor ventilación pulmonar, reflejado en un aumento considerable del VE. Todo ello podría ser debido a la activación involuntaria de grupos musculares que no participan directamente en el $\mathrm{PB}$, lo cual supone un estrés mayor al sistema cardiovascular. Además, el grupo $\mathrm{ADF}$ mostró valores más bajos de eficiencia de su sistema cardiorrespiratorio con un $\mathrm{VO}_{2} / \mathrm{FC} 57 \%$ y $68 \%$ inferior (a intensidad 1 y 2 respectivamente) que el grupo Control. Este hecho se debe a que la mutación genética de la ADF provoca alteraciones en la producción energética mitocondrial, hecho relacionado en ocasiones con cardiomiopatías pero siempre con una detectada disminución de la eficiencia cardíaca (Shawn y col., 2007; Maring y Croarkin, 2007).

Respecto a la percepción subjetiva del esfuerzo realizado (PE), el grupo de ADF percibió un incremento del esfuerzo apenas del $48 \%$, cuando la potencia requerida fue duplicada (a Intensidad 2). De modo similar al grupo PC, aunque en menor cuantía, las personas con ADF parecen estar acostumbradas a realizar grandes esfuerzos durante su vida diaria, debido a la dificultad de coordinación intermuscular y en ocasiones la espasticidad, y su percepción del esfuerzo se ve disminuida.

Para las personas con ADF, el pedaleo de brazos podría ser un excelente ejercicio aeróbico que propicie el mantenimiento y la mejora de su condición física, con beneficios importantes en su sistema cardiorrespiratorio e incluso en su coordinación intermuscular. Sin embargo debe tenerse en consideración que, al igual que para las personas con PC (aunque en menor grado) las intensidades del ejercicio suelen generar respuestas cardiorrespiratorias más altas que para otras personas y podrían acercarse o sobrepasar con relativa facilidad los límites del ejercicio aeróbico saludable, de intensidad ligera o moderada. Esto puede ser especialmente peligroso para las personas con ADF debido a sus frecuentes alteraciones cardíacas. El control habitual o periódico de la respuesta del organismo al ejercicio, con dispositivos de monitorización de la FC, podría ser recomendable, al menos durante la dosificación de nuevas cargas de entrenamiento. Por supuesto, el control médico periódico así como el seguimiento de sus recomendaciones es de obligado cumplimiento. 
González-Carbonell, I.; Brizuela, G., y Romero-Ávila, J. L. (2016). Pedaleo de brazos en personas con lesión medular, parálisis cerebral o ataxia cerebelosa: Parámetros fisiológicos. RICYDE. Revista internacional de ciencias del deporte. 46(12), 359-381. http://dx.doi.org/10.5232/ricyde2016.04602

Finalmente, mientras la ADF no evolucione hasta impedir el ejercicio físico, y se pueda realizar de forma habitual, el sobrepeso no debería ser una preocupación especial, debido a su GE relativamente incrementado. Sin embargo, no debe descuidarse la ingesta calórica ya que, aunque durante la realización de ejercicio el gasto energético resulte algo más alto que el esperable, el gasto de energía diario difícilmente alcanzaría al de una persona sedentaria sin alteraciones neurológicas.

El grupo PC mostró una FC 86 \% más alta a la intensidad 1 y 72 \% más alta a la intensidad 2 que el grupo Control, acompañado también de los valores más altos de $\mathrm{VO}_{2} \mathrm{R}$ (junto al grupo ADF) y de un incremento importante de FR y VE, debido a la espasticidad y contracción de musculatura no protagonista del movimiento, que somete al organismo a un estrés más alto. La afectación del SNC causa una descoordinación muscular que les lleva a la implicación involuntaria de mucha más masa muscular de lo estrictamente necesario en el movimiento de $\mathrm{PB}$, por lo que tanto su FC como su GE son más altos que para el resto de grupos, duplicando el valor de GE del grupo Control. Estos resultados parecen diferir con los obtenidos en el estudio de Tobimatsu, Nakamura y Kusano, (1998), en el que se realizaron pruebas en PB con personas con $\mathrm{PC}$, al 75\% de la FC máxima, y no se encontraron diferencias significativas respecto al grupo control. Sin embargo, en su estudio los participantes presentaban una afectación muy leve (GMFS grado I, deambulantes e independientes en ABVD) y eran muy jóvenes, mientras que en nuestro estudio los participantes del grupo PC presentaban una afectación motriz mucho más elevada (GMFS grados IV y V).

A pesar de lo expuesto, es muy interesante destacar el trabajo de De Groot, Dallmeijer, Bessems, Lamberts y Woude, (2012) con personas adultas con PC y físicamente activas, comparando tanto la capacidad aeróbica como la fuerza muscular, respecto a un grupo de control. Sus resultados subrayan que no existen diferencias en el $\mathrm{VO}_{2} \mathrm{MÁX}$, pero la fuerza muscular de las personas con PC está limitada respecto al grupo de control. Parcialmente de acuerdo, la revisión de Hombergen, Huisstede, Streur, Stam, Slaman, Bussmann y BergEmons (2012), destaca la reducción en la fuerza muscular $(34 \%-60 \%)$, la resistencia muscular $(27 \%-52 \%)$ y la resistencia cardiorrespiratoria $(14 \%)$ en jóvenes con PC, comparado con un grupo Control.

Lo que resultó muy sorprendente en el grupo PC, es la similitud en la FC registrada a las dos diferentes intensidades de ejercicio, causa por la cual la interacción entre el factor Grupo y el factor Intensidad resultara significativa $(\mathrm{p}<0,001)$. Tras la sorpresa inicial, cabe explicarse de un modo muy simple: para las personas con PC ya supone un estímulo altamente estresante (y motrizmente complicado) moverse y realizar ejercicio. La dificultad para coordinar la acción cíclica de diferentes grupos musculares y realizar el PB es una tarea sumamente compleja para una persona con PC y alto grado de afectación motriz y, un incremento ligero o medio en la carga de trabajo, puede resultar sencillamente no medible en incrementos de FC. En el mismo sentido, y basado en la experiencia de trabajar con personas con PC, podría sugerirse que con una carga de trabajo relativamente mayor su coordinación intermuscular sea más eficiente y el ejercicio de PB resulte más fluido. De este modo, el incremento de respuesta cardíaca necesaria para compensar un aumento de la intensidad de trabajo, podría verse equilibrado con un pedaleo más fluido que necesitaría, por el contrario, algo menos de energía.

De modo similar a lo ocurrido con la FC, el VE no mostró diferencia entre ambas intensidades de ejercicio para el grupo PC, reflejando que su respuesta respiratoria al ejercicio viene determinada en mayor medida por la necesidad de coordinar el movimiento, luchando contra su propia espasticidad, más que por vencer la carga del ejercicio, al menos a 
González-Carbonell, I.; Brizuela, G., y Romero-Ávila, J. L. (2016). Pedaleo de brazos en personas con lesión medular, parálisis cerebral o ataxia cerebelosa: Parámetros fisiológicos. RICYDE. Revista internacional de ciencias del deporte. 46(12), 359-381. http://dx.doi.org/10.5232/ricyde2016.04602

las intensidades analizadas en el presente estudio. Destacando un trabajo respiratorio muy considerable, este grupo dobló el valor de VE del resto de grupos y, sin incrementar el VT con el aumento de intensidad de ejercicio, alcanzaron los valores más altos de FR: $183 \%$ a Intensidad 1 y $205 \%$ a Intensidad 2, respecto al grupo Control. Esto podría deberse a que para las personas con PC es mucho más natural y quizás más eficiente aumentar la FR que intentar llenar más los pulmones en cada respiración (VT), lo cual supondría un reto más coordinativo y complejo.

Tanto la $\mathrm{VE} / \mathrm{VO}_{2}$ como el $\mathrm{R}$ medidos para el grupo $\mathrm{PC}$, muestran los mayores valores del estudio y reflejan un esfuerzo pulmonar mayor así como una aproximación a la utilización de la vía anaeróbica de provisión de energía, al pasar a la Intensidad 2 del ejercicio (comprobado también para el grupo LMA).

Al igual que para el grupo ADF, cuando se incrementó un $100 \%$ la intensidad del ejercicio, el grupo PC sólo percibió (PE) un aumento del $48 \%$, mientras sus resultados en VE, FR y $\mathrm{VO}_{2} \mathrm{R}$ fueron las más altos entre todos los grupos. Del mismo modo que se ha explicado para el grupo ADF, su percepción de la intensidad del esfuerzo se ve disminuida.

\section{Implicaciones prácticas}

En el presente estudio se ha comprobado que el ejercicio de PB puede generar fácilmente niveles de estrés altos en el sistema cardiorrespiratorio de las personas con PC. Intensidades de ejercicio que para otras personas podrían resultar ligeras o muy ligeras, podrían para ellas superar sus límites de ejercicio aeróbico saludable, debido a sus características particulares, especialmente a la espasticidad. Esto podría ocurrir también al realizar otros tipos de ejercicio físico.

Teniendo en cuenta que una vida activa y la práctica habitual de EF es recomendado para las personas con PC, sería muy aconsejable el control habitual de la respuesta de su organismo al ejercicio, utilizando dispositivos de monitorización de la FC, teniendo en cuenta además, que su percepción al esfuerzo podría verse alterada (disminuida) y no reflejaría la intensidad real del ejercicio. Evidentemente es necesario además, un control de salud periódico que permita alertar del riesgo de accidentes cardiovasculares y/o cerebrovasculares, evitando especialmente el ejercicio de intensidad elevada.

En cuanto al balance energético, el sobrepeso no debería ser de especial preocupación para las personas con $\mathrm{PC}$ de tipo espástica. Al igual que en otros estudios, se ha comprobado que su gasto energético durante la AF (y una vida diaria moderadamente activa) es relativamente alto (el más alto en este estudio) por lo que la ingesta calórica podrá superar normalmente a la de una persona con LM cervical.

\section{Conclusiones}

De modo general se puede confirmar que el efecto que origina el ejercicio de pedaleo de brazos sobre la respuesta fisiológica de personas con diferentes desórdenes neurológicos es variado y depende del tipo y del grado de la propia afectación neurológica. La respuesta cardíaca, respiratoria y de gasto energético, durante el ejercicio de pedaleo de brazos, resulta diferente entre personas con PC, ADF y LM. Las personas con PC muestran una respuesta cardíaca, respiratoria y de gasto energético, aumentada frente al resto de grupos, mientras que las personas con LM muestran los valores más bajos, y muy próximos o similares al grupo Control. Las personas con ADF muestran respuestas cardiorrespiratorias y de gasto energético intermedias. 
González-Carbonell, I.; Brizuela, G., y Romero-Ávila, J. L. (2016). Pedaleo de brazos en personas con lesión medular, parálisis cerebral o ataxia cerebelosa: Parámetros fisiológicos. RICYDE. Revista internacional de ciencias del deporte. 46(12), 359-381. http://dx.doi.org/10.5232/ricyde2016.04602

La respuesta cardíaca, respiratoria y de gasto energético es incrementada positivamente al aumentar la intensidad del ejercicio de PB para las personas con ADF y LM. Sin embargo, para las personas con PC un incremento de la intensidad del ejercicio modifica únicamente su gasto energético y su frecuencia respiratoria.

En base a los resultados observados, puede concluirse que el ejercicio de PB genera suficiente estrés en el organismo de las personas con desórdenes neurológicos como para ser considerado un excelente método en la búsqueda del incremento en la AF habitual de estas personas y contribuir a reducir tanto el sedentarismo como los problemas de salud asociados.

En cuanto a limitaciones del presente estudio, en primer lugar, debe tenerse en consideración que no se han podido seguir las recomendaciones tradicionales sobre el trabajo en cicloergómetro y la valoración se ha realizado a una cadencia de pedaleo de $20 \mathrm{~min}^{-1}$, cadencia a la que perfectamente pueden hacerlo personas con LM, PC y ADF y alto grado de afectación.

En vistas de futuros trabajos relacionados con este, la medición complementaria de la lactacidemia podría aportar información muy relevante para comprobar la implicación de la vía anaeróbica durante la generación del trabajo, especialmente en las personas con LM y PC.

Del mismo modo, sería muy recomendable intentar ampliar el estudio incorporando un mayor número de participantes así como un número equilibrado de personas de ambos géneros en todos los grupos e incluso con subgrupos de personas con diferentes niveles de afectación neurológica.

\section{Referencias}

Adams, G.M. (2002). Exercise physiology: Laboratory manual. Boston: McGraw-Hill Interamericana.

Bastian, A.J. (1997). Mechanisms of ataxia. Physical Therapy, 77(6), 672-5.

Bauman, W.A. (2009). The potential metabolic consequences of cerebral palsy: Inferences from the general population and persons with spinal cord injury. Developmental Medicine and Child Neurology, 51, 64-78. http://dx.doi.org/10.1111/j.1469-8749.2009.03430.x

Bax, M.; Goldstein, M.; Rosenbaum, P.; Leviton, A.; Paneth, N.; Dan, B., \& Damiano, D. (2005). Proposed definition and classification of cerebral palsy. Developmental Medicine and Child Neurology, 47(8), 571-576. http://dx.doi.org/10.1017/S001216220500112X

Brenes, G.; Dearwater, S.; Shapera, R.; LaPorte, R.E., \& Collins, E. (1986). High density lipoprotein cholesterol concentrations in physically active and sedentary spinal cord injured patients. Archives of Physical Medicine and Rehabilitation, 67(7), 445-450.

Brizuela, G.; Sinz, S.; Aranda, R., y Martínez, I. (2010). Efecto del pedaleo de brazos sobre el sistema cardiorrespiratorio de las personas con tetraplejia. RICYDE. Revista internacional de ciencias del deporte, 21(6), 297-310. http://dx.doi.org/10.5232/ricyde2010.02104

Borg, G. (1982) Psychophysical bases of perceived exertion. Medicine and Science in Sports and Exercise, 14, 377-381. http://dx.doi.org/10.1249/00005768-198205000-00012

Buchholz, A.C., \& Pencharz, P.B. (2004). Energy expenditure in chronic spinal cord injury. Clinical Nutrition and Metabolic Care, 7(6), 635-639.

http://dx.doi.org/10.1097/00075197-200411000-00008 
González-Carbonell, I.; Brizuela, G., y Romero-Ávila, J. L. (2016). Pedaleo de brazos en personas con lesión medular, parálisis cerebral o ataxia cerebelosa: Parámetros fisiológicos. RICYDE. Revista internacional de ciencias del deporte. 46(12), 359-381. http://dx.doi.org/10.5232/ricyde2016.04602

Chamberlain, S.; Shaw, J.; Rowland, A.; Wallis, J.; South, S.; Nakamura, Y., \& Williamson, R. (1988). Mapping of mutation causing Friedreich's ataxia to human chromosome 9. Nature, 334(6179), 248-250.

http://dx.doi.org/10.1038/334248a0

Collins; E.G.; Gater, D.; Kiratli, J.; Butler, J.; Hanson, K. \& Langbein, W.E. (2010). Energy cost of physical activities in persons with spinal cord injury. Medicine and Science in Sports and Exercise, 42(4); 691-700. http://dx.doi.org/10.1249/MSS.0b013e3181bb902f

Conger, S.A., \& Bassett, D.R. (2011). A compendium of energy costs of physical activities for individuals who use manual wheelchairs. Adapted Physical Activity Quarterly, 28(4), 310-325.

Dallmeijer, A.J., \& van der Woude, L.H.V. (2001). Health related functional status in men with spinal cord injury: Relationship with lesion level and endurance capacity. Spinal Cord, 39, 577-583.

http://dx.doi.org/10.1038/sj.sc.3101215

Dallmeijer, A.J.; Zentgraaff, D.B.; Zijp, N.I., \& van der Woude, L.H.V. (2004). Submaximal physical strain and peak performance in handcycling versus handrim wheelchair propulsion. Spinal Cord, 42, 91-98.

http://dx.doi.org/10.1038/sj.sc.3101566

De Groot, S.; Dallmeijer, A.J.; Bessems, P.J.C.; Lamberts, M.L.; van der Woude, L.H.V., \& Janssen, T.W.J. (2012). Comparison of muscle strength, sprint power and aerobic capacity in adults with and without cerebral palsy. Journal of Rehabilitation Medicine, 44(11), 932.

http://dx.doi.org/10.2340/16501977-1037

Dearwater, S.R.; Laporte, R.E.; Cauley, J.A., \& Brenes, G. (1985). Assessment of physical activity in inactive populations. Medicine and Science in Sports and Exercise, 17(6), 651-654.

http://dx.doi.org/10.1249/00005768-198512000-00005

Delatycki, M.B.; Williamson, R., \& Forrest, S.M. (2000). Friedreich ataxia: An overview. Journal of Medical Genetics, 37(1), 1-8.

http://dx.doi.org/10.1136/jmg.37.1.1

Devís-Devís, J. (2001). La educación física, el deporte y la salud en el siglo XXI. Colección educación física y deporte. Alcoy: Marfil.

Ditunno, J.F.; Young, W.; Donovan, W.H., \& Creasey, G. (1994). The International standards bokklet for neurological and functional classification of spinal-cord injury. Paraplegia, 32(2), 70-80.

http://dx.doi.org/10.1038/sc.1994.13

Engel, J.M.; Schwartz, L.; Jensen, M.P., \& Johnson, D.R. (2000). Pain in cerebral palsy: The relation of coping strategies to adjustment. Pain, 88(3), 225-230. http://dx.doi.org/10.1016/S0304-3959(00)00330-4

Erikssen, G.; Liestøl, K.; Bjørnholt, J.; Thaulow, E.; Sandvik, L., \& Erikssen, J. (1998). Changes in physical fitness and changes in mortality. The Lancet, 352, 759-762.

Figoni, S.F. (1993). Exercise responses and quadriplegia. Medicine and Science in Sports and Exercise, 25, 433-441. http://dx.doi.org/10.1016/S0140-6736(98)02268-5

Fillyaw, M.J., \& Ades, P.A. (1989). Endurance exercise training in friedreich ataxia. Physical Medicine and Rehabilitation, 70(10), 786-788. 
González-Carbonell, I.; Brizuela, G., y Romero-Ávila, J. L. (2016). Pedaleo de brazos en personas con lesión medular, parálisis cerebral o ataxia cerebelosa: Parámetros fisiológicos. RICYDE. Revista internacional de ciencias del deporte. 46(12), 359-381. http://dx.doi.org/10.5232/ricyde2016.04602

Folkins, C., \& Sime, W. (1981). Physical fitness training and mental health. American Psychologist, 36, 373-389.

http://dx.doi.org/10.1037/0003-066X.36.4.373

Garber, C.E.; Blissmer, B.; Deschenes, M.R.; Franklin, B.A.; Lamonte, M.J.; Lee, M.D. et al (2011). Quantity and quality of exercise for developing and maintaining cardiorespiratory, musculoskeletal, and neuromotor fitness in apparently healthy adults: Guidance for prescribing exercise. Medicine and Science in Sports and Exercise, 43(7), 1334-1359.

http://dx.doi.org/10.1249/MSS.0b013e318213fefb

Goosey-Tolfrey V.L., \& Sindall, P. (2007). The effects of arm crank strategy on physiological responses and mechanical efficiency during submaximal exercise. Journal of Sports Sciences, 25(4), 453-460.

http://dx.doi.org/10.1080/02640410600702883

Haisma, J.A.; van der Woude, L.H.V.; Stam, H.J.M.; Bergen, M.P.; Sluis, T.A., \& Bussmann, J.B.J. (2006). Physical capacity in wheelchair-dependent persons with a spinal cord injury: A critical review of the literature. Spinal Cord, 44, 642-652. http://dx.doi.org/10.1038/sj.sc.3101915

Hicks, A.L.; Martin, K.A.; Ditor, D.S.; Latimer, A.E.; Craven, C.; Bugaresti, J., \& McCartney, N. (2003). Long-term exercise training in persons with spinal cord injury: Effects on strength, arm ergometry performance and psychological wellbeing. Spinal Cord, 41(1), 34-43.

http://dx.doi.org/10.1038/sj.sc.3101389

Hicks, A.L.; Martin, K.A.; Pelletier, C.A.; Ditor, D.S.; Foulon, B., \& Wolfe, D. L. (2010). The effects of exercise training on physical capacity, strength, body composition and functional performance among adults with spinal cord injury: A systematic review. Physical Medicine and Rehabilitation, 91(5), 729-733.

Hjeltnes, N. (1986). Cardiorespiratory capacity in tetra- and paraplegia shortly after injury. Scandinavian Journal of Rehabilitation Medicine, 18(2), 65-70.

Hjeltnes, N., \& Wallberg-Henriksson, H. (1998). Improved work capacity but unchanged peak oxygen uptake during primary rehabilitation in tetraplegic patients. International Medical Society of Paraplegia, 36(691), 698. http://dx.doi.org/10.1038/sj.sc.3100687

Hombergen, S.P.; Huisstede, B.M.; Streur, M.F.; Stam, H.J.; Slaman, J.; Bussmann, J.B., \& van den Berg-Emons, R.J. (2012). Impact of cerebral palsy on health-related physical fitness in adults: Sistematic review. Archives of Physical Medicine and Rehabilitation, 93(5), 871-881.

http://dx.doi.org/10.1016/j.apmr.2011.11.032

Ilg, W.; Synofzik, M.; Broetz, D.; Burkard, S.; Giese, M.A., \& Schoels, L. (2009). Intensive coordinative training improves motor performance in degenerative cerebellar disease. Neurology, 73(22), 1823-1830.

http://dx.doi.org/10.1212/WNL.0b013e3181c33adf

Jahnsen, R.; Villien, L.; Egeland, T.; Stanghelle, J.K., \& Holm, I. (2004). Locomotion skills in adults with cerebral palsy. Clinical Rehabilitation, 18(3), 309-316. http://dx.doi.org/10.1191/0269215504cr735oa

Johnson, R.K.; Hildreth, H.G.; Contompasis, S.H., \& Goran, M.I. (2005). Total energy expenditure in adults with cerebral palsy as assessed by doubly labeled water. Medicine and Science in Sports and Exercise, 37(3), 347-353. 
González-Carbonell, I.; Brizuela, G., y Romero-Ávila, J. L. (2016). Pedaleo de brazos en personas con lesión medular, parálisis cerebral o ataxia cerebelosa: Parámetros fisiológicos. RICYDE. Revista internacional de ciencias del deporte. 46(12), 359-381. http://dx.doi.org/10.5232/ricyde2016.04602

Krum, H.; Howes, L.G.; Brown, D.J.; Ungar, G.; Moore, P.; McNeil, J.J., \& Louis, W.J. (1992). Risk factors for cardiovascular disease in chronic spinal cord injury patients. International Medical Society of Paraplegia, 30, 381-388. http://dx.doi.org/10.1038/sc. 1992.87

Lawson, J.A. (2006). Comparative quantification of health risks. Global and regional burden of disease attributable to selected major risk factors. Ottawa: Canadian Public Health Association.

Lidal, I.B.; Snekkevik, H.; Aamodt, G.; Hjeltnes, N.; Stanghelle, J.K., \& BieringSorensen, F. (2007). Mortality after spinal cord injury in Norway. Journal of Rehabilitation Medicine, 39(2), 145-151. http://dx.doi.org/10.2340/16501977-0017

Malina, R.M., \& Bouchard, C. (1991). Somatic growth. En: Growth, maduration, and physical activity. pp. 39-64. Champaign: Human Kinetics.

Maring, J.R., \& Croarkin, E. (2007). Presentation and progression of friedreich ataxia and implications for physical therapist examination. Physical Therapy, 87(12), 16871696. http://dx.doi.org/10.2522/ptj.20060232

Mariotti, C.; Fancellu, R., \& Di Donato, S. (2005). An overview of the patient with ataxia. Journal of Neurology, 252(5), 511-518.

http://dx.doi.org/10.1007/s00415-005-0814-z

Monroe, M.B.; Tataranni, P.A.; Pratley, R.; Manore, M.M.; Skinner, J.S., \& Ravussin, E. (1998). Lower daily energy expenditure as measured by chamber in subjects with spinal cord injury compared with control subjects. American Journal of Clinical Nutrition, 68(6), 1223-1227.

Murphy, K.P.; Molnar, G.E., \& Lankasky, K. (1995). Medical and functional status of adults with cerebral palsy. Developmental Medicine and Child Neurology, 37(12), 1075-1084.

http://dx.doi.org/10.1111/j.1469-8749.1995.tb11968.x

Mutch, L.; Alberman, E.; Hagberg, B.; Kodama, K., \& Perat, M.V. (1992). Cerebral palsy epidemiology: Where are we now and where are we going?. Developmental Medicine and Child Neurology, 34(6), 547-551.

http://dx.doi.org/10.1111/j.1469-8749.1992.tb11479.x

Nash, S.M.; Bilsker, S.; Marcillo, A. E.; Isaac, S. M.; Botelho, L. A.; Klose, J.K.; Green, B.A.; Rountree, M.T., \& Shea, D.J. (1991). Reversal of adaptive left ventricular atrophy following electrically-stimulated exercise training in human tetraplegics. Paraplegia, 29, 590-599. http://dx.doi.org/10.1097/01.NPT.0000282514.94093.c6

Nash, S.M. (2005). Exercise as a health-promoting activity following spinal cord injury. Journal of Neurological Physical Therapy, 29(2), 87-106. http://dx.doi.org/10.1038/sc.1991.87

Pelletier, C.A.; Jones, G.; Latimer-Cheung, A.E.; Warburton, D.E., \& Hicks, A.L. (2013). Aerobic capacity, orthostatic tolerance, and exercise perceptions at discharge from inpatient spinal cord injury rehabilitation. Achieves of Physical Medicine and Rehabilitation, 10(94), 2013-2019.

http://dx.doi.org/10.1016/j.apmr.2013.05.011

Peterson, M.D.; Gordon, P.M., \& Hurvitz, E.A. (2013 a). Chronic disease risk among adults with cerebral palsy: The role of premature sarcopoenia, obesity and sedentary behaviour. Obesity Reviews, 14(2), 171-182.

http://dx.doi.org/10.1111/j.1467-789X.2012.01052.x 
González-Carbonell, I.; Brizuela, G., y Romero-Ávila, J. L. (2016). Pedaleo de brazos en personas con lesión medular, parálisis cerebral o ataxia cerebelosa: Parámetros fisiológicos. RICYDE. Revista internacional de ciencias del deporte. 46(12), 359-381. http://dx.doi.org/10.5232/ricyde2016.04602

Peterson, M.D.; Lukasik, L.; Muth, T.; Esposito, P.; Haapala, H.; Gordon, P. M., \& Hurvitz, E.A. (2013 b). Recumbent cross-training is a feasible and safe mode of physical activity for significantly motor-impaired adults with cerebral palsy. Achieves of Physical Medicine and Rehabilitation, 94, 401-407. http://dx.doi.org/10.1016/j.apmr.2012.09.027

Rajan, S.; McNeely, M.J.; Warms, C., \& Goldstein, B. (2008). Clinical assessment and management of obesity in individuals with spinal cord injury: A review. Journal of Spinal Cord, 31(4), 361-372.

Rimmer J.H. \& Rowland J.L. (2008). Health promotion for people with disabilities: implications for empowering the person and promoting disability-friendly environments. American Journal of Lifestyle Medicine, 2, 409-420. http://dx.doi.org/10.1177/1559827608317397

Ristow, M.; Giannakidou, E.; Hebinck, J.; Busch, K.; Vorgerd, M.; Kotzka, J., \& MuellerWieland, D. (1998). An association between NIDDM and a GAA trinucleotide repeat polymorphism in the X25/frataxin (Friedreich's ataxia) gene. Diabetes, 47(5), 851854. http://dx.doi.org/10.2337/diabetes.47.5.851

Schuhfried, O.; Mittermaier, C.; Jovanovic, T.; Pieber, K., \& Paternostro-Sluga, T. (2005). Effects of whole-body vibration in patients with multiple sclerosis: A pilot study. Clinical Rehabilitation, 19(8), 834-842.

http://dx.doi.org/10.1191/0269215505cr919oa

Shawn, A.G.; Calum A.M.; Kusai, A.; Sims, K.B.; Schmahmann, J.D.; Kardan, A., \& Fischman, A.J. (2007). Myocardial blood flow and oxygen consumption in patients with Friedreich's ataxia prior to onset or cardiomyopathy. Coronary Artery Disease, 18(1), 15-22.

http://dx.doi.org/10.1097/01.mca.0000236289.02178.60

Taylor, N.F.; Dodd, K.J., \& Larkin, H. (2004). Adults with cerebral palsy benefit from participating in a strength training programme at a community gymnasium. Disability and Rehabilitation, 26(19), 1128-1134.

http://dx.doi.org/10.1080/09638280410001712387

Thorpe, D. (2009). The role of fitness in health and disease: Status of adults with cerebral palsy. Developmental Medicine and Child Neurology, 51, 52-58.

http://dx.doi.org/10.1111/j.1469-8749.2009.03433.x

Tobimatsu, Y.; Nakamura, R., \& Kusano, S. (1998). Cardiorespiratory endurance in people with cerebral palsy measuring with arm ergometer. Physical Medicine and Rehabilitation, 79, 991-993.

http://dx.doi.org/10.1016/S0003-9993(98)90099-5

Toung, T.; Chang, Y.; Williams, M.; Crain, B.; Traystman, R., \& Bhardwaj, A. (2004). Experimental spinal cord ischemia: Model characterization and improved outcome with arterial hypertension. Critical Care Medicine, 32(6), 1346-1351. http://dx.doi.org/10.1097/01.CCM. 0000128562.80108.61

Valent, L.J.M.; Dallmeijer, A.J.; Houdijk, H.; Slootman, J.H.; Janssen, T.W., \& van der Woude, L.H.V. (2009). Effects of hand cycle training on physical capacity in individuals with tetraplegia: A clinical trial. Physical Activity and Therapy, 89, 10511060. http://dx.doi.org/10.2522/ptj.20080340

Washburn, R.A., \& Figoni, S.F. (1999). High density lipoprotein cholesterol in individuals with spinal cord injury: The potential role of physical activity. Spinal Cord, 37(10), 685-695.

http://dx.doi.org/10.1038/sj.sc.3100917 
González-Carbonell, I.; Brizuela, G., y Romero-Ávila, J. L. (2016). Pedaleo de brazos en personas con lesión medular, parálisis cerebral o ataxia cerebelosa: Parámetros fisiológicos. RICYDE. Revista internacional de ciencias del deporte. 46(12), 359-381. http://dx.doi.org/10.5232/ricyde2016.04602

West, C.R.; Mills, P., \& Krassioukov, A.V. (2012). Influence of the neurological level of spinal cord injury on cardiovascular outcomes in humans: A meta-analysis. Spinal Cord, 50(7), 484-492.

http://dx.doi.org/10.1038/sc.2012.17

Yamasaki, M.; Irizawa, M., \& Komura, T. (1992). Daily energy expenditure in active and inactive persons with spinal cord injury. Journal of Human Ergology, 21, 125-133. 